\title{
FACILITATING HUFF FOR AIRWAY CLEARANCE AND TO RELIEVE DYSPNEA IN SUBJECTS WITH COPD
}

\author{
Deepika Burman ${ }^{* 1}$, Sneha Ghuman ${ }^{2}$, Varoon Jaiswal ${ }^{3}$, Snehal Ghodey ${ }^{4}$. \\ ${ }^{* 1}$ B.P.TH student, MAEER's Physiotherapy College, Pune, Maharashtra, India. \\ ${ }^{2}$ Assistant Professor, MAEER's Physiotherapy College, Pune, Maharashtra, India. \\ ${ }^{3}$ Associate Professor, MAEER's Physiotherapy College, Pune, Maharashtra, India. \\ ${ }^{4}$ Principal, MAEER's Physiotherapy College, Pune, Maharashtra, India.
}

\section{ABSTRACT}

Introduction: Chronic obstructive pulmonary disease (COPD), is a lung disease characterised by chronic obstruction of lung airflow that interferes with normal breathing and is not fully reversible. Secretions produced in the respiratory tract are cleared by muco-ciliary transport, cephalad airflow bias, and cough which then helps in relieving dyspnea. There is need to find an alternate cost effective, easy technique which can help clearing the secretions and relieve dyspnea. An intervention like huff training could help better in clearance of airways. Removal of secretions will also facilitate in relieving dyspnea. Forced expiration against resistance has demonstrated better sputum production and reduced the level of dyspnea.

Procedure: Permission from the Institutional Ethical committee was taken before starting the study. Two groups were made, group A (Experimental group) and group B (Control group) comprising of 10 subjects each. All the subjects were explained about the study and a informed written consent was taken from them. Subjects were randomly allocated. 24 hours sputum expectoration was collected in the calibrated sputum mug \& it was measured at the end of 24 hours. The level of dyspnea was measured by using VAS scale for breathlessness pre and post 24 hours.

Results: Lung clearance was better with therapist made huffing device and amount of sputum was statistically significant (analysed using Mann Whitney's Test) and there was improvement in dyspnea (Level of Dyspnoea was statistically analysed using Wilcoxin's Matched Paired Test).

Conclusion: The study concludes that HUFF given through the therapist made device was more effective than the traditional HUFF in clearance of airway secretions as well as in relieving dyspnea in subjects with Acute Exacerbation of Chronic Obstructive Pulmonary Disease.

KEY WORDS: COPD, Therapist- made device, Amount of sputum, Level of Dyspnea, VAS scale for Breathlessness.

Address for correspondence: Deepika Burman, NITI Merchant Society, Bunglow No 15, Besides Vedanta Academy, Station Road, Malavali-410405, Pune, Maharashtra, India.

E-Mail: deepikaburman4@gmail.com

\section{Access this Article online}

Quick Response code

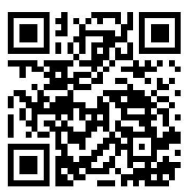

DOI: $10.16965 /$ ijpr.2017.130
International Journal of Physiotherapy and Research

ISSN 2321- 1822

www.ijmhr.org/ijpr.html

Received: 08-03-2017

Peer Review: 09-03-2017

Revised: None
Accepted: 03-04-2017

Published (O): 20-05-2017

Published (P): 11-06-2017

\section{INTRODUCTION}

Chronic Obstructive Pulmonary Disease (COPD) is not one single disease but an umbrella term used to describe chronic lung diseases that cause limitations in lung airflow. Long-term exposure to other lung irritants-such as air pollution, chemical fumes, or dust-also may contribute to COPD [1,2]. According to World Health Organization estimates, 65 million people have moderate to severe COPD [3]. Emphysema, chronic bronchitis \& bronchial asthma are the common conditions that make up COPD [4]. 
Acute exacerbation of COPD also known as acute exacerbations of chronic bronchitis (AECB) is a sudden worsening of COPD. Some of the more common symptoms of advancing COPD and acute exacerbation include: 1.Ongoing cough. 2. Cough that is accompanied by excessive mucus production, often referred to as a "smoker's cough". 3. Chest tightness. 4. Shortness of breath which is made worse with physical activity. 5 . Wheezing inability to catch breath or speak. 6. Confusion or excessive sleepiness. 6. Rapid heartbeat. 7. Blue or grey fingernails or lips. 8. Increase in mucus production. 9. Change in colour of mucus to tan, yellow, green, or bloody. 10. Fever.

Some of the initial treatment may include one or more of bronchodilator oxygen therapy, systemic glucocorticosteroids (oral or inhaler). Chest physiotherapy is an important aspect \& plays a pivotal role in both obstructive and restrictive conditions. Secretions produced in the respiratory tract are cleared by mucociliary transport, cephalad airflow bias, and cough. Techniques have been developed to optimize expiratory flow and promote airway clearance. In diseased lung, increased secretion viscosity and volume, dyskinesia of the cilia, and ineffective cough combine to reduce the ability to clear secretions, and may increase exacerbations and infections [5].

A variety of breathing manoeuvres have been developed, refined, and used to assist patients in mobilizing secretions from the lower respiratory tract. However, though the rationale may be sound, there is a paucity of evidence supporting such strategies. ACBT improves pulmonary function and airway clearance similar to conventional CPT. The therapist made huffing device made from the PVC pipe will help for loosening and removal of secretions as the balloon tied at the other end of pipe will act as a resistance against active exhalation and also produce a backflow which will assist in clearance of airway secretions. There is a need to find an alternate inexpensive, easy technique for lung clearance and relieving dyspnea.

An intervention like HUFF training could help better to come out of exacerbation early by clearing the secretions and relieving dyspnea, and also control these symptoms even after the patient is out of exacerbation than the conventional techniques. Very few studies are conducted to find out the effective technique for lung clearance other than conventional method and the conventional devices available are very costly. Hence this study was conducted to have an effective lung clearance with the help of low cost effective therapist made device [4].

The aim of the study was to facilitate HUFF for airway clearance and to relieve dyspnea in subjects with acute exacerbation of COPD. And the objective was to 1 . Find the effect of huffing through therapist-made huffing device on sputum volume and level of dyspnea in subjects with acute exacerbation of COPD. 2. And to compare between traditional huffing and huffing through therapist-made huffing device.

\section{MATERIALS AND METHODS}

It was an Experimental study, type of sampling was Simple random sampling. Study setting was rural hospital with Physiotherapy set-up

Sample size: 20 (10 in each group)

Inclusion Criteria: 1.Patients with Acute exacerbations of COPD. 2. Patients having productive cough. 3. Patients willing to participate in the study.

Exclusion Criteria: 1.Cardiac Conditions Simulating COPD and Any other restrictive respiratory conditions with similar symptoms. 2 . Patients with neurological conditions. 3.Patients who are not co-operative. 4. Patients Not willing to participate in the study.

Materials Used: 1 . Therapist made huffing device with perforated balloon 2. Calibrated Sputum Mug 3.VAS scale for Breathlessness. 4. Stethoscope 5.Pen and paper.

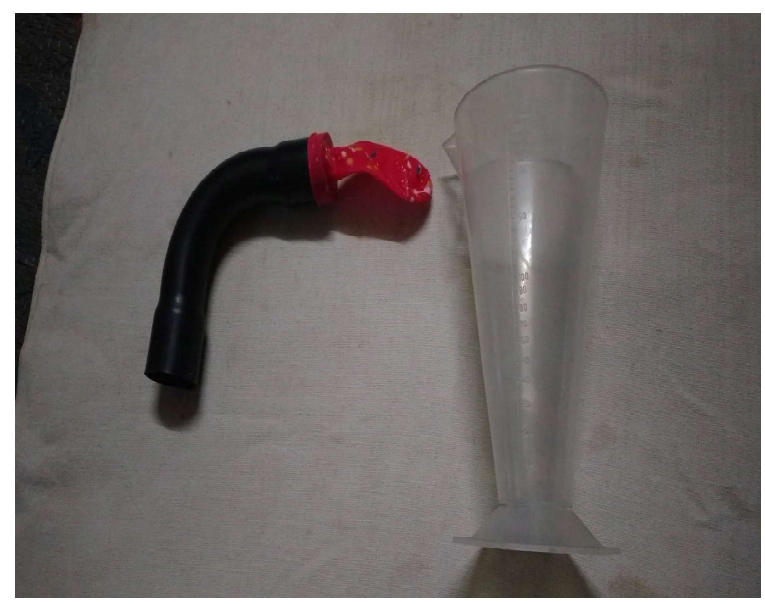


The huffing device was designed by Dr. Varoon $C$ Jaiswal (PT). The huffing device was made up of a PVC pipe, of 1 inch $(2.5 \mathrm{cms})$ diameter and a perforated balloon was attached to one end and from the other end the subject (in sitting position) was asked to blow into the pipe so as the balloon rises, the rising of balloon gave a visual feedback to the subjects. Two groups were made, group A and group B comprising of 10 subjects each. All the subjects were explained about the survey and a informed written consent was taken from them.

Procedure: Subjects were randomly allocated into each group, Group A was the Experimental group whereas group $B$ was the Control group. Group A was given Huff through the therapist made huffing device whereas group B was given Traditional Huff. Subject was asked to blow into the pipe for 1 minute as many times as he can (should stop if they experience breathlessness or any severe discomfort). Subject was told to repeat this for around 4-5 times every 2-3 hours and this has to be done around 15-20 times for 24 hours. In both the groups subject was asked to dispose the Sputum in the provided calibrated sputum mug and the amount of secretions in the sputum beaker was collected after $24 \mathrm{hrs}$. Also the level of dyspnea, measured by VAS scale for breathlessness was taken pre and post the study for each patient.

\section{RESULTS AND STATISTICAL ANALYSIS}

The statistical analysis was done using GraphPadInstat.

Amount of sputum collected over a period of 24hours in Experimental and Control group was statistically analysed using Mann Whitney's Test. VAS pre and post over a period of 24hours in the Experimental group was statistically analysed using the Wilcoxin's Matched Paired Test.

VAS pre and post over 24hours in the Control group was statistically analysed using the Wilcoxin's Matched Paired Test.

Post VAS in the Experimental and Control group over a period of 24 hours was statistically analysed using the Mann Whitney's Test.

The mean difference of VAS in Experimental and Control group over a period of 24 hours was statistically analysed using Mann Whitney's Test.
Table 1: Amount of Sputum collected in Experimental and Control group.

\begin{tabular}{|c|c|c|c|c|}
\hline & $\begin{array}{c}\text { Experimental } \\
\text { group }\end{array}$ & $\begin{array}{c}\text { Control } \\
\text { group }\end{array}$ & P value & U value \\
\hline Mean & 92 & 35 & \multirow{2}{*}{0.0002} & 99 \\
\hline SD & 26.583 & 10.801 & & \\
\hline
\end{tabular}

Considered extremely significant

Table 2: Post VAS in Experimental and Control Group.

\begin{tabular}{|c|c|c|c|c|}
\hline & $\begin{array}{c}\text { Experimental } \\
\text { group }\end{array}$ & $\begin{array}{c}\text { Control } \\
\text { group }\end{array}$ & P value & U value \\
\hline Mean & 1.18 & 1.74 & \multirow{2}{*}{0.1121} & 71.5 \\
\hline SD & 0.6779 & 0.7792 & & \\
\hline
\end{tabular}

Considered not significant

Table 3: Mean difference of VAS in Experimental and Control group.

\begin{tabular}{|c|c|c|c|c|}
\hline & $\begin{array}{c}\text { Experimental } \\
\text { group }\end{array}$ & $\begin{array}{c}\text { Control } \\
\text { group }\end{array}$ & P value & U value \\
\hline Mean & 2.47 & 0.86 & \multirow{2}{*}{0.001} & 94 \\
\hline SD & 0.9546 & 0.4033 & & \\
\hline
\end{tabular}

Considered very significant

\section{DISCUSSION}

The study was designed to find out the effect of huffing through the therapist made huffing device on the clearance of airway secretions and the level of dyspnea. The study was done on 20 subjects amongst whom 10 subjects were included into experimental group whereas 10 subjects were included in the control group randomly. The subjects in the experimental group were given HUFF through the therapist made huffing device whereas the subjects in the control group were given Traditional HUFF i.e. without the therapist made huffing device. In both the groups the protocol was given and the sputum was collected over a period of 24 hours, also dyspnea was measured pre and post the treatment protocol in both the groups.

On the basis of the above statistical result there were improved results seen in the experimental group than the control group in terms of sputum as well as the level of dyspnea. Studies have shown that in patients with acute exacerbation of COPD, if the airways clearance is done first and mucus is cleared up it helps the patient come out of the exacerbation and also it helps for the prognosis of the patients and makes them asymptomatic. So it was very important to find out the ways for clearance of airway secretions, 
and also they should be easily available and patient friendly and hence this study was done. At the same time it was equally important to find out such technique in which patients can perform the clearance techniques by themselves without the help of the therapist or when the therapist is not there.

Patients with Chronic Obstructive Pulmonary Diseases and chronic Bronchitis often produce a large amount of mucus. If the mucus is allowed to collect in the airways, breathing may become difficult and infection may occur. During the period of exacerbations the sputum becomes both more copious and more vicious and thus more difficult to expectorate. Huffing is a technique that helps move mucus from the lungs. It moves the sputum from the smaller airways to larger airways. It involves taking a deep breath in, holding it and exhaling. Breathing in and holding it enables air to get behind the mucus and separates it from the lung wall so it can be blown out.

As huffing causes less dynamic compression in lungs and does not causes large airway collapse so hence it can be used easily in patients with COPD. But sometimes it becomes difficult for Therapist to teach huffing to the patients, so with our device it was easier to teach huffing to the patients and also the sputum expectorated through our therapist made huffing device was more than the traditional huff. The huffing given through the therapist made device must have proved more effective than the huffing given without the therapist made device because when the patient is given huff through the device it gives patient a visual feedback of the inflating balloon as they blow into the pipe, hence therapist informing patient the correct huffing technique. Also this device is therapist and patient friendly i.e. when once the patient is taught the correct technique of doing it the patient can perform it by himself even when the therapist is not present.

\section{CONCLUSION}

The study concludes that HUFF given through the therapist made device was more effective than the traditional HUFF in clearance of airway secretions and relieveing in dyspnea in subjects with Acute Exacerbation of Chronic Obstructive Pulmonary Disease.
Clinical Significance: We can use this device for airway clearance in patients who are in Acute Exacerbation of COPD so that they can come out of this exacerbation and become symptom free.

Limitations: Female's were not included in the study. Drugs during protocol period. Subjects were not strictly supervised during 24 hours of treatment protocol.

\section{Conflicts of interest: None}

\section{REFERENCES}

[1]. Chronic respiratory disease April 2006 3; 83-91. Falk $P$, Eriksen A-M, Kølliker K, Andersen J B. Relieving dyspnea with an inexpensive and simple method in patients with severe chronic airflow limitation; Eur J Respir Dis 1985;66:181-6.

[2]. Advances in airway clearance technologies for COPD Medscape multispecialty 2013;7(6)673-685.

[3]. Physiotherapy practice in management of patients with bronchiectasis and chronic obstructive pulmonary disease. Alfred Hospital, University of Melbourne, School of Physiotherapy.

[4]. API Textbook of Medicine; Sidharth .N. Shaha.

[5]. Hie T, Pas BG, Roth RD, Jensen WM. Huff coughing and airway patency Respir care 1979;24(8):710-713.

[6]. Airway clearance techniques for bronchiectasis Annemarie L Lee1,2,3 Angela Burge Anne E Holland School of Physiotherapy, The University of Melbourne, Carlton, Australia.

[7]. Chest physiotherapy in chronic obstructive pulmonary disease : forced expiratory technique combined with either postural drainage or positive expiratory pressure L Olseni, B Midgren, Y Hörnblad, P Wollmer - Respiratory medicine, 1994 - Elsevier

[8]. Efficacy of physical therapy methods in airway clearance patients with chronic obstructive pulmonary disease Nowobilski1,2, Tomasz $W^{3}$ och1,2, Maciej $P^{3}$ aszewski3, Andrzej Szczeklik1

[9]. Pulmonary rehabilitation. American Thoracic Society. Am J RespirCrit Care Med.1999;159(5 Pt 1):16661682.

[10]. Sleigh MA.Ciliary function in transport of mucus. Eur J Respir Dis 1983;128(Pt 1):287-292.

[11]. Thompson B, Thompson HT, Forced expiratory exercises in asthma and their effect on FEV1,NZ J Physiotherapy 1963;3:19-21.

[12]. Partridge C, Pryor J, Webber B. Characteristics of the forced expiratory technique Physiotherapy 1989;75:193-194.

[13]. Van Weinden CM, Visser A, Hop W, Sterk P J, Beckers $\mathrm{S}$, deJongste JC. Effects of flutter and PEP mask physiotherapy on symptoms and lung function in children with cystic fibrosis . EurRespir J 1998;12(1 ):143147.

[14]. Pontifex E Williams MT, Lunn R Parsons D. The effects of huffing and directed coughing on energy expenditure in young asymptomatic subjects Aust $\mathrm{J}$ Physiotherapy 2002;48(3):209-213.

[15]. Holland AE, Button BM. Is there a role of airway clearance techniques in Chronic Obstructive Pulmonary Disease and Chron Respir Dis 2006;3(2):83-91. 